\title{
Qualidade de vida em mulheres submetidas à mastectomia comparada com aquelas que se submeteram à cirurgia conservadora: uma revisão de literatura
}

\author{
Quality of life of women recovering from breast cancer \\ after being subjected to mastectomies compared with those \\ who had conservative surgery: a review of the literature
}

Juliana Machado Majewski ${ }^{1}$

Aline Daniela Fernandes Lopes ${ }^{1}$

Tárcia Davoglio ${ }^{1}$

José Carlos de Carvalho Leite ${ }^{2}$

${ }^{1}$ Grupo de pesquisa em

Psicologia da Saúde,

Programa de Pós-

Graduação em Psicologia,

Faculdade de Psicologia,

Pontifícia Universidade

Católica do Rio Grande do

Sul (PUC/RS). Av. Ipiranga

6687/ Prédio 11/937,

Partenon. 90619-900 Porto

Alegre RS.

danielafl@yahoo.com.br

${ }^{2}$ Universidade do Vale do

Rio dos Sinos

\begin{abstract}
This study reviews the literature on the quality of life (QoL) of women with breast cancer who have been subjected to mastectomy, compared with those who had conservative surgery. Eight random controlled trials were selected. The studies were compared with respect to the moment quality of life was assessed (whether during or after treatment for breast cancer), the measurement tools of quality of life used, and also the methodology and results achieved. The results of four studies suggest a stronger negative impact in the QoL of mastectomized women; the other four studies showed no difference between the groups in terms of QoL. Objective measurements of quality of life may help identify potentially critical situations of daily life and assist in planning actions to promote health among women who have been subjected to breast cancer surgery

Key words Breast cancer, Mastectomy, Quality oflife
\end{abstract}

Resumo Este estudo revisa a literatura sobre a qualidade de vida $(\mathrm{QV})$ de mulheres com câncer de mama submetidas à mastectomia comparadas àquelas que realizaram cirurgia conservadora. A seleção final resultou em oito ensaios clínicos randomizados. Os estudos foram comparados quanto ao momento em que a $Q V$ foi avaliada, se durante ou após o tratamento, quanto aos instrumentos que mensuraram a $\mathrm{QV}$, e quanto à metodologia e resultados. Os resultados de quatro estudos apontam para maior impacto negativo na QV em mulheres mastectomizadas; outros quatro estudos não evidenciam diferenças na $Q V$ entre os grupos que passaram pelos dois tipos de intervenção. Medidas objetivas de QV poderão ajudar a identificar situações potencialmente difíceis da vida diária e auxiliar no planejamento de ações de promoção da saúde de mulheres que passaram por cirurgia para câncer de mama.

Palavras-chave Câncer de mama, Mastectomia, Qualidade de vida 


\section{Introdução}

O câncer é uma das principais causas de morte no mundo. A Organização Mundial da Saúde $(\mathrm{OMS})^{1}$ estima que o número de mortes por câncer no mundo deverá crescer $45 \%$ entre 2007 e 2030 , saltando de 7,9 milhões para 11,5 milhões de óbitos anuais e o número de casos novos deverá aumentar de 11,3 milhões para 15,5 milhões anuais no mesmo período. Entre os tipos de câncer de maior incidência mundial encontra-se o câncer de mama, que é o mais prevalente entre as mulheres, envolvendo em sua etiologia tanto fatores genéticos quanto ambientais ainda não totalmente conhecidos ${ }^{2}$.

No Brasil, as estimativas do Instituto Nacional do Câncer ${ }^{3}$ para 2010/2011 apontam para o risco de 49 novos casos de câncer de mama a cada 100 mil mulheres, sendo que a região sudeste é a área de maior incidência $(65 / 100.000)$, seguida pela região sul do país $(64 / 100.000)$. O número de diagnósticos da doença em estágio inicial é ainda considerado insuficiente, pois, em torno de $80 \%$ dos casos novos que são diagnosticados apresentam tumores em estágio avançado (III ou IV), fazendo com que a cirurgia invasiva seja a alternativa terapêutica mais recomendada. Portanto, a detecção precoce, envolvendo o rastreamento e o diagnóstico em fases incipientes, é considerada prioridade no enfrentamento do câncer de mama; e a reabilitação física, psíquica e social devem ser abordadas como problema de saúde pública, cuja efetividade repercute em melhores resultados no tratamento ${ }^{4,5}$.

Ainda hoje, o diagnóstico de câncer é percebido como sendo "uma sentença de morte", mesmo com o avanço da medicina em relação ao tratamento e com o aumento de informações veiculadas pela mídia. Durante todo o processo da doença são vividos, pela paciente e sua família, sentimentos de intenso sofrimento e ansiedade. Entre as mulheres portadoras de câncer de mama é muito comum o temor à mutilação, os preconceitos sociais, o medo da morte e do surgimento de linfedemas, além de sentimentos depressivos e de desvalorização social ${ }^{6,7}$. O câncer de mama e seu tratamento podem levar a mulher a alterações na sua autoimagem, além de perda funcional e mudanças a nível psíquico, emocional e social ${ }^{8}$. A doença muitas vezes proporciona uma perda de papéis relacionadas ao trabalho, à família e à sexualidade 9 .

$O$ tratamento do câncer de mama pode envolver intervenções locais ou sistêmicas, utilizadas de forma independente ou concomitante. A cirurgia e a radioterapia podem ser entendidas como formas de tratamento local e que visam à remoção ou à destruição do tumor em uma determinada área do corpo. A quimioterapia, a hormonioterapia e a terapia biológica são técnicas utilizadas para combater a doença de forma sistêmica, buscando controlar ou destruir o câncer na extensão de todo o organismo ${ }^{10,11}$.

Os processos cirúrgicos são os mais utilizados na terapêutica do câncer de mama, priorizando as demandas oncológicas essenciais para a manutenção da vida e, somente após, as questões estéticorreparadoras. Assim, há duas classificações: a cirurgia conservadora e a mastectomia. As cirurgias conservadoras retiram apenas parte da glândula mamária que contém o tumor e normalmente não causam prejuízo na sobrevida total, embora aumentem de forma não muito significativa a taxa de recidiva local do tumor. A mastectomia, por sua vez, é um procedimento que visa à retirada total da glândula mamária, com o objetivo de reduzir a incidência e melhorar a expectativa de vida de mulheres pertencentes a populações consideradas de alto risco ${ }^{10}$, sendo quase sempre inevitável em fases adiantadas da doença.

A mulher mastectomizada enfrenta a difícil realidade de conviver com a amputação da mama. Embora o tratamento possa ser determinante para sua sobrevivência, gera muitos temores. O mais frequente temor da mulher mastectomizada ainda é a fantasia de não ser mais atraente sexualmente, já que a mama, simbolicamente, se associa a identidade feminina e a sua ausência representaria uma limitação estética e psíquica muito significativa ${ }^{12,13}$. A barganha mama versus vida durante muito tempo foi imposta pelas formas de tratamento de uma época na qual as questões psicológicas relacionadas à autoimagem não eram consideradas como parte do tratamento ${ }^{4}$. Atualmente, considerando a relevância da qualidade de vida $(\mathrm{QV})$, sempre que possível são oferecidos tratamentos que preservem a mama e diminuam significativamente $o$ sofrimento psicológico e social da mulher.

Nos últimos anos, considerando o impacto que o diagnóstico e o tratamento do câncer de mama geram na vida da mulher, tem se dado maior ênfase às pesquisas envolvendo medidas de QV. Questionários para medir QV têm sido utilizados em investigações clínicas, tanto para descrever grupos de pacientes como para obter o resultado de avaliações com relação à efetividade das intervenções propostas na área da saúde. São apontadas como medidas facilitadoras para o 
reconhecimento de problemas funcionais e emocionais nem sempre detectáveis na avaliação clínica convencional, oportunizando a melhor monitoração e comunicação das pacientes com a equipe médica ${ }^{14}$.

A Organização Mundial da Saúde define QV como "a percepção do indivíduo de sua posição na vida, no contexto da cultura e sistemas de valores nos quais vive, em relação aos seus objetivos, expectativas, padrões e preocupações" ${ }^{\prime 1}$. No entanto, há duas tendências na conceituação do termo: (a) QV entendida de forma mais genérica; (b) QV descrita com relação à saúde. Qualidade de Vida Relacionada à Saúde (QVRS) é um subconjunto do termo amplo QV e recebe esta classificação por incluir os domínios físico, psicológico e social que possuem relação com a saúde $\mathrm{e}^{14,16,17}$.

Acredita-se que medidas quantitativas de QV possam guiar estratégias de intervenções terapêuticas e auxiliar na definição de ações que priorizem a promoção da saúde ${ }^{8}$. Profissionais de saúde e pacientes tentam avaliar as influências do tratamento oncológico para a cura ou o controle da doença, visando compreender os desfechos do tratamento bem sucedido, na visão da própria paciente ${ }^{18}$, já que a QV se dá a partir de uma avaliação subjetiva dos processos da doença e da recuperação ${ }^{19}$.

Várias técnicas e instrumentos foram desenvolvidos para avaliar as funções do indivíduo, a situação de saúde e a $Q V$, podendo ou não ser específicos para determinada patologia ${ }^{20}$. Entre as medidas específicas para avaliar QV em portadoras de câncer de mama destacam-se: Breast Cancer Module (BR-23) ${ }^{21}$, Functional Assessment of Cancer Therapy of Breast Cancer (FACT$B)^{22}$ e European Organization for Research and Treatment of Cancer Quality of Life Questionnaire (EORTC QLQ-C30) ${ }^{23}$. Entre as medidas mais usadas para avaliar QV em geral estão as seguintes: Medical Outcomes Study 36-Item Short-Form Healty Survey (SF-36) ${ }^{24}$ e World Health Organization Quality of Life (WHOQOL100 ou WHOQOL-bref) $)^{8,25,26}$.

Este estudo revisou ensaios clínicos randomizados sobre intervenções cirúrgicas realizadas em portadoras de câncer de mama. O objetivo principal foi avaliar as evidências de associação entre mastectomia e mudança na QV dessas mulheres.

\section{Método}

A busca dos artigos para este estudo foi realizada nas bases de dados indexadas Medline, Lilacs, Scielo e Psycinfo, utilizando-se descritores tanto em língua portuguesa quanto inglesa. Objetivou-se selecionar estudos que avaliaram a QV de mulheres com câncer de mama, submetidas ao tratamento de cirurgia conservadora ou mastectomia.

A sintaxe da pesquisa foi dividida em 4 subgrupos. O primeiro teve como referência o tipo de tratamento, sendo utilizados os seguintes descritores: mastectomy/mastectomia, mastectomized/mastectomizadas, breast cancer surgery/ cirurgia de câncer de mama, radiotherapy/radioterapia, chemoterapy/quimioterapia e hormone therapy/hormonioterapia. O segundo subgrupo foi classificado a partir do diagnóstico utilizando os termos breast cancer/câncer de mama, mammary tumors/tumor mamário, breast carcino$\mathrm{ma} /$ carcinoma mamário e breast neoplasms/neoplasia mamária. O terceiro utilizou os descritores Quality of life/Qualidade de Vida e Health Related Quality of life/Qualidade de Vida Relacionada à saúde. O último subgrupo incluiu termos relacionados ao delineamento dos estudos, utilizando como descritores randomized controlled trial/ensaio clínico randomizado, controlled clinical trial/ensaio clínico controlado, evaluation studies/estudo de avaliação, clinical trials/ensaios clínicos, clinical trial/ensaio clínico, random allocation/atribuição aleatória, double blind method/método duplo-cego, single-blind method/ método simples-cego, research design/projeto de pesquisa, comparative study/estudo comparativo, follow-up studies/estudos de acompanhamento e prospective studies/estudos prospectivos. Os descritores dos quatro subgrupos foram combinados entre si para a realização da busca, incluindo artigos publicados de 1980 até 30 de maio de 2010.

Foram identificados 316 artigos, sendo que destes 262 pertenciam à base de dados Medline e 54 à Psycinfo. Nas bases Lilacs e Scielo não foram encontrados artigos relacionados ao objetivo da busca. Foram considerados para a inclusão apenas artigos com delineamento de Ensaio Clínico Randomizado que abordavam a QV de mulheres submetidas à mastectomia em comparação com a cirurgia conservadora da mama. Foram excluídos artigos que não mencionavam a QV relacionada à mastectomia e à cirurgia conservadora, estudos que faziam comparação com a eficiência de medicamentos utilizados na quimioterapia sobre a QV, estudos que focavam apenas a reabi- 
litação de pacientes com a prática de atividades físicas, pesquisas que não fossem Ensaios Clínicos Randomizados, artigos que não falavam sobre o câncer de mama ou citavam apenas intervenções específicas com relação à quimioterapia $\mathrm{e}$ à radioterapia e os estudos de revisão.

Foram selecionados para a avaliação final oito artigos de interesse para o estudo, sendo sete pertencentes à base de dados Medline e um pertencente à Psycinfo. Após a leitura cuidadosa do texto completo, estes artigos foram analisados quanto ao momento que se avaliou a $\mathrm{QV}$, se durante ou após o tratamento, os instrumentos que mensuraram a QV, a metodologia, os resultados e a possibilidade de comparação entre os estudos.

\section{Resultados}

Dos oito artigos incluídos na revisão, sete foram realizados com amostras de origem ocidental, predominando nestas as de descendência europeia, havendo apenas um estudo com amostra oriental. O momento que se avaliou a QV nestes artigos variou entre primeiro mês e segundo ano após a intervenção cirúrgica. Vários instrumentos foram utilizados para mensurar a $\mathrm{QV}$, não havendo um consenso no uso dos mesmos, sendo que alguns utilizaram questionários próprios, elaborados pelos autores. A Tabela 1 mostra uma síntese dos artigos, considerando os instrumentos utilizados, os objetivos de se avaliar QV, o momento em que se avaliou a mesma e os principais resultados encontrados.

Entre os artigos selecionados, os primeiros foram publicados na década de 80 , sendo o primeiro realizado por Haes e Welvaar $\mathrm{t}^{27}$ utilizando um questionário sugerido por Meyerowitz ${ }^{28}$ que faz uma análise do impacto do câncer de mama na esfera psicossocial das mulheres. Neste mesmo estudo, também foram utilizadas escalas sobre a satisfação conjugal, impacto sobre a imagem corporal e o medo de recidiva e morte. O objetivo principal foi comparar aspectos psicossociais, sexuais, imagem corporal, medo de recidiva e morte, através da avaliação da QV, 11 meses após a cirurgia, comparando mulheres que passaram pela mastectomia com aquelas que passaram por cirurgia conservadora. Foram selecionadas 41 participantes, das quais 39 (18 mastectomizadas e 21 cirurgia conservadora) preencheram aos questionários enviados pelos autores, levando-os a concluir que a imagem corporal das pacientes após a mastectomia estava severamente mais afetada do que na cirurgia conservadora.
Referente ao medo de recorrência da doença e morte, não houve diferença significativa entre os grupos, mas estavam proporcionalmente relacionados à idade dos pacientes. Não houve diferença no que diz respeito ao funcionamento sexual.

Em um novo estudo, Haes et al. ${ }^{29}$ utilizaram os mesmos questionários do estudo anterior ${ }^{27}$, acrescidos do Rotterdan Symptom ChecklistRSCL $^{30,31}$, além de novas escalas formuladas para avaliar os aspectos psicológicos, sexuais, imagem corporal, medo da recidiva e morte e Qualidade de Vida Global. Neste estudo foram encontrados os mesmos resultados do estudo publicado em 1985, sugerindo que, do ponto de vista psicológico, o tratamento de conservação da mama deveria, sempre que possível, ser priorizado em mulheres de todas as idades. Assim como no estudo anterior, 41 pacientes foram selecionadas e 39 responderam ao questionário 11 meses após a intervenção, com o diferencial de que 6 meses após este período os pacientes receberam novamente os questionários e apenas 34 (17 mastectomizadas e 17 cirurgia conservadora) das 39 responderam, pois uma paciente morreu e quatro recusaram-se a participar. Nesse estudo, observou-se que a QV e os sofrimentos físico e psicológico melhoraram significativamente com o tempo, após 11 meses, tanto no grupo de mulheres mastectomizadas quanto naquele de cirurgia conservadora.

Kemeny et al..$^{32}$ utilizaram a escala Brief Sympton Inventory- $\mathrm{BSI}^{33}$ e questionários elaborados especificamente para o estudo. Após seis meses de tratamento, investigaram as consequências na sexualidade das mulheres, focando quais eram as vantagens ou as desvantagens associadas à amputação da mama em comparação com a conservação da mesma, alterações da imagem corporal, medo de recidiva e de morte e o trauma de ver a cirurgia. $\mathrm{O}$ estudo selecionou $83 \mathrm{mu}$ lheres, das quais 52 preencheram o questionário no primeiro envio (27 mastectomizadas e 25 cirurgia conservadora). $\mathrm{Na}$ segunda tentativa de envio, apenas 37 das 52 pacientes responderam ao questionário. Os resultados encontrados sugerem que mulheres não mastectomizadas possuíam níveis de satisfação mais positivos com relação à sexualidade e à imagem corporal do que as que passaram pelo procedimento. A preocupação com relação à recidiva foi menor nas pacientes que passaram pela cirurgia conservadora. O trauma de ver o resultado da cirurgia foi muito maior em pacientes mastectomizadas.

Identificar os problemas das mulheres após a mastectomia total ou a cirurgia conservadora 
Tabela 1. Síntese dos estudos que avaliaram a QV

\begin{tabular}{|c|c|c|c|c|}
\hline Autor/Ano & $\begin{array}{c}\text { Instrumentos } \\
\text { de } Q V^{*}\end{array}$ & $\begin{array}{c}\text { Itens de QV } \\
\text { avaliados }\end{array}$ & $\begin{array}{l}\text { Período da } \\
\text { coleta }\end{array}$ & Resultados \\
\hline Haes et al. $1985^{27}$ & $\begin{array}{l}\text { Questionário } \\
\text { sugerido por } \\
\text { Meyerowitz; } \\
\text { Escalas } \\
\text { desenvolvidas pelos } \\
\text { autores. }\end{array}$ & $\begin{array}{l}\text { Aspectos } \\
\text { sexuais, imagem } \\
\text { corporal, medo } \\
\text { de recidiva e } \\
\text { morte }\end{array}$ & $\begin{array}{l}11 \text { meses } \\
\text { após a } \\
\text { cirurgia }\end{array}$ & $\begin{array}{l}\text { Imagem corporal severamente danificada } \\
\text { após a mastectomia. Grupos não diferem } \\
\text { quanto aos temores de recidiva e morte. Não } \\
\text { houve diferença quanto ao funcionamento } \\
\text { sexual. }\end{array}$ \\
\hline Haes et al. $1986^{29}$ & $\begin{array}{l}\text { Questionário } \\
\text { sugerido por } \\
\text { Meyerowitz; } \\
\text { Rotterdam } \\
\text { Symptom } \\
\text { Checklist (RSCL); } \\
\text { Escalas } \\
\text { desenvolvidas pelos } \\
\text { autores. }\end{array}$ & $\begin{array}{l}\text { Aspectos } \\
\text { psicológicos, } \\
\text { sexuais, imagem } \\
\text { corporal, medo } \\
\text { de recidiva e } \\
\text { morte }\end{array}$ & $\begin{array}{l}11 \text { e } 18 \\
\text { meses após } \\
\text { a cirurgia }\end{array}$ & $\begin{array}{l}\text { Imagem corporal severamente prejudicada } \\
\text { após a mastectomia. Medo de recorrência de } \\
\text { câncer não foi relacionado ao tipo de } \\
\text { tratamento. A QV melhorou e o sofrimento } \\
\text { psicológico e físico diminuiu em ambos os } \\
\text { grupos com o tempo. }\end{array}$ \\
\hline $\begin{array}{l}\text { Kemeny et al. } \\
1988^{32}\end{array}$ & $\begin{array}{l}\text { Brief Symptom } \\
\text { Inventory (BSI); } \\
\text { Escalas } \\
\text { desenvolvidas pelos } \\
\text { autores. }\end{array}$ & $\begin{array}{l}\text { Consequências } \\
\text { na sexualidade } \\
\text { das mulheres }\end{array}$ & $\begin{array}{l}6 \text { e } 14 \text { meses } \\
\text { após } \\
\text { acirurgia }\end{array}$ & $\begin{array}{l}\text { Imagem corporal e sexualidade prejudicadas } \\
\text { após a mastectomia. O trauma da visualização } \\
\text { do resultado cirúrgico e medo da recidiva } \\
\text { foram maiores em pacientes } \\
\text { mastectomizadas. }\end{array}$ \\
\hline Ganz et al. $1992^{41}$ & $\begin{array}{l}\text { Functional Living } \\
\text { Index-Cancer } \\
\text { (FLIC); Cancer } \\
\text { Rehabilitation } \\
\text { Evaluation System } \\
\text { (CARES); } \\
\text { Karnofsky } \\
\text { Performance } \\
\text { Status (KPS); } \\
\text { Profile of Mood } \\
\text { States (POMS); } \\
\text { Global Adjustment } \\
\text { to Illness Scale } \\
\text { (GAIS) }\end{array}$ & $\begin{array}{l}\text { Humor e } \\
\text { ajustamento } \\
\text { psicológico }\end{array}$ & $\begin{array}{l}1,4,7 \text { e } 13 \\
\text { meses após a } \\
\text { cirurgia }\end{array}$ & $\begin{array}{l}\text { Não foram encontradas diferenças } \\
\text { significativas na QV, humor ou ajuste } \\
\text { psicossocial. Não houve diferenças } \\
\text { significativas nas necessidades de reabilitação } \\
\text { global. O grupo de mastectomia radical } \\
\text { apresentou dificuldades maiores de problemas } \\
\text { com a imagem corporal e do vestuário. }\end{array}$ \\
\hline $\begin{array}{l}\text { Curran et al. } \\
1998^{16}\end{array}$ & $\begin{array}{l}\text { Breve questionário } \\
\text { sobre qualidade de } \\
\text { vida. }\end{array}$ & $\begin{array}{l}\text { Consequências } \\
\text { para imagem } \\
\text { corporal, temor } \\
\text { de recidiva e } \\
\text { satisfação com o } \\
\text { tratamento e } \\
\text { resultados } \\
\text { estéticos. }\end{array}$ & $\begin{array}{l}2 \text { anos após } \\
\text { a cirurgia }\end{array}$ & $\begin{array}{l}\text { Vantagem significativa para terapia de } \\
\text { conservação quanto à imagem corporal. Não } \\
\text { houve diferença quanto ao temor de recidiva. } \\
\text { Em ambos os grupos as pacientes mais jovens } \\
\text { denotaram maior temor de recidiva. } \\
\text { Satisfação com o tratamento maior entre } \\
\text { àquelas que passaram por cirurgia } \\
\text { conservadora. }\end{array}$ \\
\hline Haes et al. $2003^{39}$ & $\begin{array}{l}\text { Questionário } \\
\text { formulado pelos } \\
\text { pesquisadores } \\
\text { abrangendo } \\
\text { diferentes } \\
\text { domínios da QV }\end{array}$ & $\begin{array}{l}\text { Sobrevida e } \\
\text { preferência de } \\
\text { tratamento em } \\
\text { pacientes idosos } \\
\text { com mais de } 70 \\
\text { anos, com } \\
\text { câncer de } \\
\text { mama precoce }\end{array}$ & $\begin{array}{l}2 \text { meses após } \\
\text { a cirurgia e } \\
12 \text { meses } \\
\text { após o } \\
\text { estudo }\end{array}$ & $\begin{array}{l}\text { Não houve diferença significativa com relação } \\
\text { à fadiga, funcionamento emocional, medo de } \\
\text { reincidência, apoio social, funcionamento } \\
\text { físico, atividades de lazer e sobrevida. } \\
\text { Pacientes tratadas com cirurgia conservadora } \\
\text { relataram maior benefício em relação à } \\
\text { imagem corporal. }\end{array}$ \\
\hline
\end{tabular}


Tabela 1. continuação

\begin{tabular}{|c|c|c|c|c|}
\hline Autor/Ano & $\begin{array}{c}\text { Instrumentos } \\
\text { de } Q V^{\star}\end{array}$ & $\begin{array}{c}\text { Itens de QV } \\
\text { avaliados }\end{array}$ & $\begin{array}{l}\text { Período da } \\
\text { coleta }\end{array}$ & Resultados \\
\hline $\begin{array}{l}\text { Chang et al. } \\
2007^{40}\end{array}$ & $\begin{array}{l}\text { Avaliação funcional do } \\
\text { Chronic Illness - } \\
\text { Therapy-Breast, } \\
\text { traditional Chinese } \\
\text { version } 4 \text { (FACIT-B) e } \\
\text { FACIT-G }\end{array}$ & QV & $\begin{array}{l}12 \text { meses } \\
\text { após a } \\
\text { cirurgia }\end{array}$ & $\begin{array}{l}\text { As mulheres não relataram pior QV, mas elas } \\
\text { eram mais propensas a se preocupar com sua } \\
\text { imagem corporal resultante. Metade teria } \\
\text { escolhido um procedimento menos extenso. } \\
\text { As mulheres que escolheram o tipo de } \\
\text { tratamento tiveram maior probabilidade de } \\
\text { satisfação com os resultados. }\end{array}$ \\
\hline $\begin{array}{l}\text { Skrzypulec et al. } \\
2007^{34}\end{array}$ & $\begin{array}{l}\text { Quality of Life - Breast } 23 \\
\text { (QL - BR 23); Life } \\
\text { Satisfaction Index (LSI); } \\
\text { Impact of Event Scale } \\
\text { (IES); Hospital Anxiety } \\
\text { and Depression Scale } \\
\text { (HADS); e Sexuality } \\
\text { Scale. }\end{array}$ & $\begin{array}{l}\text { Desconforto } \\
\text { pós-cirúrgico, } \\
\text { insatisfação } \\
\text { com a vida, } \\
\text { nível de } \\
\text { depressão e } \\
\text { ansiedade. }\end{array}$ & $\begin{array}{l}\text { Diferentes } \\
\text { momentos } \\
\text { após a } \\
\text { cirurgia } \\
\text { entre julho } \\
\text { de } 2004 \text { e } \\
\text { abril de } 2005\end{array}$ & $\begin{array}{l}\text { O stress pós-cirúrgico está mais fortemente } \\
\text { associado à manifestação de sintomas } \\
\text { depressivos, de ansiedade e de insatisfação } \\
\text { com a vida em mulheres mastectomizadas, } \\
\text { comparado àquelas que passaram por cirurgia } \\
\text { conservadora. }\end{array}$ \\
\hline
\end{tabular}

${ }^{*} \mathrm{QV}$ : Qualidade de Vida

da mama, com relação a fatores que aumentam a intensidade de desconforto e que consequentemente afetam a QV, foram os objetivos do estudo de Skrzypulec et al. ${ }^{34}$. Este estudo utilizou-se das seguintes escalas: QL-BR ${ }^{23,21}$ Life satisfaction Index - LSI ${ }^{35}$, Impact of Event Scale - IES ${ }^{36}$, Hospital Anxiety And Depression Scale - $\mathrm{HADS}^{37} \mathrm{e}$ Sexuality Scale ${ }^{38}$ e selecionou 550 mulheres (433 mastectomizadas e 117 cirurgia conservadora). Foram excluídas da pesquisa 30 mulheres por não se adequarem aos critérios de inclusão que eram mastectomia total devido ao câncer de mama e preenchimento do consentimento de participação e do questionário. Por fim, restaram 403 mulheres com mastectomia radical. No grupo controle composto por mulheres com cirurgia conservadora, restaram 91 pacientes, pois as demais não preencheram os critérios de inclusão. Ao final, 494 mulheres fizeram parte da pesquisa. O estudo concluiu que mulheres mastectomizadas possuíam intensidade significativa de stress pós-traumático diretamente relacionado com os níveis de depressão e ansiedade, o que acarretou no funcionamento biopsicossocial inferior em comparação com mulheres submetidas à cirurgia conservadora.

Dos artigos selecionados, três deles - Haes et al. ${ }^{39}$, Chang et al. ${ }^{40}$ e Ganz et al. ${ }^{41}$ - não encontraram diferenças significativas entre os resultados obtidos. No estudo de Haes et $\mathrm{al}^{39}$, foram avaliadas 136 pacientes (65 mastectomizadas e 71 cirurgia conservadora) com mais de 70 anos. Foi aplicado um questionário formulado pelos pesquisa- dores com 36 itens que abrangeram diferentes domínios da QV. Neste estudo, não foram encontradas diferenças no que diz respeito à fadiga, ao funcionamento emocional, ao medo de reincidência, ao apoio social, ao funcionamento físico, a atividades de lazer e à sobrevida entre os grupos.

O FACT-B e o Functional Assessment of Cancer Therapy (FACT-G) ${ }^{42}$ foram utilizados por Chang et al. ${ }^{40}$ em um estudo que avaliou a QV e a preferência de tratamento de 220 mulheres com câncer de mama orientais do norte de Taiwan (157 mastectomizadas e 63 cirurgia conservadora). Os resultados apontaram que as mulheres mastectomizadas não apresentaram uma pior QV do que as que receberam cirurgia conservadora, em contrapartida, estavam mais propensas a se preocupar com sua imagem corporal. As mulheres que escolheram o tipo de tratamento que realizaram tiveram maior probabilidade de satisfação com os resultados.

A pesquisa realizada por Ganz et al. ${ }^{41}$ também não obteve diferenças significativas entre os resultados. Foram selecionadas 425 pacientes com câncer de mama, das quais 179 não cumpriam os critérios de elegibilidade do estudo. A partir do conjunto de 246 indivíduos elegíveis, 127 se recusaram a participar, oito não completaram os quatro exames e duas pacientes receberam posteriormente mastectomia segmentar. As 109 participantes que preencherem todos os critérios (57 mastectomizadas e 52 cirurgia conservadora) foram o foco desta pesquisa. Estes autores utilizaram diversas escalas (Functional Living Index-Cancer - 
FLIC $^{43}$, Cancer Rehabilitation Evaluation System - CARES ${ }^{44,45}$, Karnofsky Performance Status KPS $^{46}$, Profile of Mood States - POMS ${ }^{47}$ e Global Adjustment to Illness Scale - GAIS ${ }^{48}$ e teve como objetivo avaliar a QV, o humor, a necessidade de reabilitação global e o ajuste psicológico. Encontraram significativa melhora do humor para ambos os grupos durante um ano de follow-up. Embora nos estudos de Haes et al. ${ }^{39}$, Chang et al. ${ }^{40} \mathrm{e}$ Ganz et al. ${ }^{41}$, não se tenha encontrado diferenças significativas na QV, é importante ressaltar que houve piora na autoimagem em mulheres mastectomizadas nas três pesquisas.

$\mathrm{O}$ artigo de Curran et al. ${ }^{16}$ objetivou descrever a experiência da EORTC- BCCG enfocando, sobretudo, a imagem corporal, a medida de recorrência da doença, a satisfação com o tratamento e os resultados estéticos, estes a julgar tanto pelo paciente quanto pelo médico. Utilizou-se um breve questionário sobre $\mathrm{QV}$ com escalas multi-item e com duas unidades individuais em 278 mulheres (127 mastectomizadas e 151 cirurgia conservadora). Os autores chegaram a resultados indicativos de que a terapia de conservação tem vantagem sobre o outro tipo de intervenção. Não houve diferença significativa entre o nível de medo de reincidência nos dois tipos de tratamento, porém, o medo da recorrência variou conforme a idade das pacientes. Com relação à satisfação com o tratamento, as que passaram pela terapia conservadora apresentaram resultado positivo.

\section{Discussão}

A primeira dificuldade observada na comparação dos estudos selecionados foi referente à conceituação e à definição de $\mathrm{QV}$, a qual não apresentou consenso entre os autores. Por ter se popularizado, a expressão QV transcende o conceito científico da área da saúde, sendo utilizada em diferentes contextos, com significados diversos ${ }^{8}$. Deste modo, com as variações na definição do construto, a escolha dos instrumentos de mensuração para fins de pesquisa sofre vieses e prejuízos na sistematização, tendo implicações tanto no que se refere à coleta quanto ao registro $\mathrm{e}$ interpretação dos dados. Com isso, nesta revisão, a comparação entre os resultados encontrados nos diversos estudos evidenciou limitações, pois, a maioria criou questionários próprios, portanto, não comparáveis entre si.

Além disso, poucos estudos clínicos randomizados foram realizados com este tema, chamando a atenção o fato de que os artigos selecionados são majoritariamente com amostras de origem europeia, sendo apenas um de origem oriental. Além disso, não foram encontrados estudos na língua portuguesa e com amostras brasileiras. Acredita-se que isto esteja também relacionado ao fato de que poucos instrumentos que medem QV em pacientes com câncer estejam adaptados e validados para a população brasileira.

A maioria dos estudos utilizou instrumentos enviados por correspondência pelos pesquisadores aos pacientes, para serem autoaplicados. Desse modo, pretendiam avaliar, a partir da visão do paciente, as consequências dos diferentes tratamentos sobre a sua QV. Esta abordagem considerou a QV como algo subjetivo ao sujeito, sendo que somente ele próprio poderia descrever o impacto da doença e do tratamento sobre a sua vida. No entanto, questionários autoaplicáveis são suscetíveis a erros de interpretação das questões, bem como, podem ser fortemente influenciados por fatores como a escolaridade e as capacidades cognitivas, tais como, compreensão, análise e síntese e memória. Além disso, as perdas amostrais podem ser bem elevadas na coleta não presencial, pois, a adesão ao estudo é condicionada, quase que exclusivamente, pela própria iniciativa do participante.

Todos os estudos analisaram a QV depois do procedimento cirúrgico, variando do primeiro mês até dois anos após a intervenção cirúrgica, conforme cada autor. Os objetivos de avaliar a QV diferiram entre os estudos, sendo que os principais itens avaliados referiam-se aos aspectos psicossociais, aspectos sexuais, autoimagem, humor, medo da recidiva e morte. A imagem corporal das mulheres mastectomizadas foi o item que teve pior índice em todos os estudos analisados. Mulheres que escolhiam o tipo de tratamento tinham maior probabilidade de satisfação com os resultados, juntamente com aquelas que passaram pela cirurgia de conservação. Porém, não houve diferenças entre os grupos no que se refere aos aspectos ligados a vida sexual destas pacientes.

A QV tende a melhorar com o passar do tempo, por isso há relevância na aplicação dos instrumentos em diferentes momentos após a intervenção, a fim de acompanhar as mudanças. Os estudos apontaram indícios que levam a pensar que o momento mais crítico para a QV se refere aos primeiros meses após a cirurgia. Portanto, são recomendadas intervenções voltadas para as questões de $\mathrm{QV}$ ao longo de todo processo, desde o diagnóstico até a reabilitação.

Os resultados da maioria dos estudos analisados apontaram que houve piora na QV de mu- 
lheres que realizaram mastectomia em comparação com as que realizaram cirurgia de conservação da mama. Esses dados corroboram a relevância de se priorizar a cirurgia conservadora ao invés da mastectomia, sempre que possível. No entanto, três dos oito artigos selecionados referiram não haver diferenças significativas nos níveis de QV entre estas mulheres. Dentre eles, um investigou a $\mathrm{QV}$ de pacientes idosas ${ }^{39} \mathrm{e}$, segundo os autores, paradoxalmente, as pacientes idosas, algumas vezes, apresentam até melhora na $\mathrm{QV}$ após passar pelos procedimentos oncológicos.

As pacientes incluídas nos estudos revisados totalizaram 1407 mulheres (901 mastectomizadas e 506 cirurgia conservadora). Em sua maioria, apresentavam câncer de mama em estágio inicial (I e II), onde as chances de reconstrução pós-cirurgia são muito maiores. Este fato pode ter contribuído para que o impacto na QV fosse menos evidente. Estudos ${ }^{49}$ indicam que 45,3\% dos novos casos de câncer no Brasil, entre os anos de 1995-2002, foram diagnosticados quando os tumores já estavam em estágios avançados (III e IV) nos quais a mastectomia é quase sempre indispensável. Assim, a importância da detecção precoce do câncer de mama é reafirma$\mathrm{da}$, viabilizando em geral tratamentos menos invasivos e dolorosos, tanto fisicamente quanto emocionalmente.

\section{Conclusões}

Os estudos revisados serviram de base para investigar as diferenças existentes na $\mathrm{QV}$ entre mulheres com câncer de mama submetidas à mastectomia e aquelas que não passaram por tal procedimento. Constatou-se que existe influência das modalidades de intervenções cirúrgicas ao câncer de mama sobre a QV, embora em três dos estudos isto não tenha ficado bem caracterizado.

No que diz respeito à imagem corporal, a influência da mastectomia foi unânime para a percepção negativa deste aspecto nos estudos analisados em comparação com a cirurgia conservadora, sendo um resultado previsível diante da amputação. É provável que esta percepção negativa encubra ou associe-se ao processo de luto subjacente, relacionado à perda da mama e de sua representação simbólica, impostas pela mastectomia. Estudos ${ }^{50}$ apontam que o luto é um fator de grande repercussão em eventos de vida como este, o qual pode estar sendo subdiagnosticado, embora suas consequências sejam fortemente sentidas pela paciente.

Com relação aos temores de recidiva e morte não houve diferenças significativas entre os dois grupos, embora exista uma maior propensão ao temor de recidiva em pacientes mais jovens e mastectomizadas, implícita talvez a própria gravidade do tumor e o impacto traumático da doença. Estas mulheres, portanto, podem ser as mais beneficiadas com intervenções psicológicas voltadas para estes aspectos.

Por outro lado, segundo os estudos, os fatores que influenciam a $\mathrm{QV}$ tendem a diminuir seu impacto com o passar do tempo, reduzindo também o sofrimento físico e psicológico da maioria das mulheres. Pode-se inferir que a QV global é mais afetada nos primeiros meses após o início do tratamento oncológico, em ambos os grupos de portadoras de câncer de mama analisados nesta revisão. Esta constatação empírica é valiosa para respaldar a introdução precoce de medidas de apoio e de enfrentamento, nas etapas em que a QV apresenta-se mais afetada. Um dos estudos, porém, sugere que em mulheres mais velhas a QV pode ser positivamente influenciada pela doença, independentemente das intervenções terapêuticas realizadas. É possível que este fato esteja associado à mudança de valores gerada pela enfermidade, a qual pode mobilizar desejos de aproveitar melhor e mais intensamente os momentos de prazer e de bem-estar.

Acredita-se que a inclusão de medidas de QV nas pesquisas científicas sobre o câncer de mama seja relevante. Medidas quantitativas de QV podem guiar estratégias de enfrentamento de fatores que potencializam o desconforto e o sofrimento, no intuito de minimizá-los ou contornálos, favorecendo a recuperação física e emocional da mulher. No entanto, esta revisão apontou a falta de instrumentos padronizados para avaliar a $\mathrm{QV}$ em pesquisas, as múltiplas definições atribuídas ao construto e os poucos estudos com metodologia de estudo clínico randomizado como fatores que limitaram a comparação entre os resultados dos estudos. 


\section{Referências}

1. World Health Organization (WHO). World Cancer Report, 2008. Lyon: International Agency for Research on Cancer; 2009.

2. Leite RC, Oliveira C, Ribeiro L. Câncer de mama: prevenção e tratamento. Rio de Janeiro: Prestígio; 2002.

3. Brasil. Ministério da Saúde (MS). Instituto Nacional de Câncer (INCA). Estimativa 2010: incidência de câncer no Brasil. Rio de Janeiro: Instituto Nacional de Câncer (INCA); 2009.

4. Biazús JV. Rotinas em cirurgia conservadora da mama. Porto Alegre: Artmed; 2000.

5. Machado SM, Sawada NO. Avaliação da qualidade de vida de pacientes oncológicos em tratamento quimioterápico adjuvante. Texto \& contexto enferm 2008; 17(4):750-757.

6. Carvalho CSU. A necessária atenção à família do paciente oncológico. Rev Bras Cancerol 2008; 54(1):87-96.

7. Souza MGG, Santo FHE. O olhar que olha o outro: um estudo com familiares de pessoas em quimioterapia antineoplásica. Rev Bras Cancerol 2008; 54(1):31-41.

8. Makluf ASD, Dias RC, Barra AA. Avaliação da qualidade de vida em mulheres com câncer de mama. Rev Bras Cancerol 2006; 52(1):49-58.

9. Redivo LB, Werlang BSG, Muller MC. Qualidade de vida em mulheres que procuram atendimento ginecológico. Psicologia, Saúde \& Doenças 2008; 9(1): 113-129.

10. Frasson Al, Zerwes FP. Câncer de mama. In: Azevedo DR, Barros MCM, Muller MC, organizadores. Psicooncologia e interdisciplinaridade: uma experiência na educação à distância. Porto Alegre: EDIPUCRS; 2004. p. 95-108.

11. Gebri LH, Kemp C, Nazario ACP, Ribalta JCL. Câncer de mama. In: Forones NM, Jesus-Garcia Filho R, Tadokoro H, Freire CAR, organizadores. Guia de oncologia. Barueri: Manole; 2005. p. 69-88.

12. Bergamasco RB, Ângelo M. O sofrimento de descobrir-se com câncer de mama: como o diagnóstico é experienciado pela mulher. Rev Bras Cancerol 2001; 47(3):227-287.

13. Almeida RA. Impacto da mastectomia na vida da mulher. Rev SBPH 2006; 9(2):99-113.

14. Belasco AGS, Sesso RCC. Qualidade de vida: princípios, focos de estudo e intervenções. In: Diniz DP, Schor N, organizadores. Guia de qualidade de vida. Barueri: Manole; 2006. p. 1-10.

15. The WHOQOL Group. The World Health Organization Quality of Life Assessment (WHOQOL): position paper from the World Health Organization. Soc Sci Med 1995; 41(10):1403-1410.
16. Curran D, van Dongen JP, Aaronson NK, Kiebert G, Fentiman IS, Mignolet F, Bartelink H. Quality of life of early-stage breast cancer patients treated with radical mastectomy or breast-conserving procedures: results of EORTC trial 10801. Eur J Cancer 1998; 4(3):307-314

17. Sales CACC, Paiva L, Scandiuzzi D, Anjos ACY. Qualidade de vida de mulheres tratadas de câncer de mama: funcionamento social. Rev Bras Cancerol 2001; 47(3):263-272.

18. Ventafridda V, Scaffidi E, Sbanoto A, Goldhirsch A. Atendimento de apoio e avaliação da qualidade de vida. In: União internacional contra o câncer (UICC). Manual de oncologia clínica. $8^{a}$ ed. São Paulo: Wiley; 2006. p. 835-850.

19. Segre M, Ferraz FC. O conceito de saúde. Rev Saude Publica 1997; 31(5):538-542.

20. Gillis T. Reabilitação do paciente com câncer. In: União internacional contra o câncer (UICC). $\mathrm{Ma}$ nual de oncologia clínica. $8^{\text {a }}$ ed. São Paulo: Wiley; 2006.

21. Sprangers MA, Groenvold M, Arraras JI, Franklin J, Te Velde A, Muller M, Franzini L, Williams A, de Haes HC, Hopwood P, Cull A, Aaronson NK. The european organization for research and treatment of cancer breast cancer-specific quality-of-life questionnaire module: first results from a three-country field study. J Clin Oncol 1996; 14(10):2756-2768.

22. Brady MJ, Cella DF, Mo F, Bonomi AE, Tulsky DS, Lloyd SR, Deasy S, Cobleigh M, Shiomoto G. Reliability and validity of the functional assessment of cancer therapy-breast quality of life instrument. $J$ Clin Oncol 1997; 15(3):974-986.

23. Aaronson NK, Ahmedzai S, Bergman B, Bullinger M, Cull A, Duez NJ, Filiberti A, Flechtner H, Fleishman SB, de Haes JC, Stein Kaasa, Klee M, Osoba D, Razavi D, Rofe PB, Schraub S, Sneeuw K, Sullivan M, Takeda F. The European Organization for research and treatment of cancer QLQ-C30: a quality-of-life instrument for use in international clinical trials in oncology. J Natl Cancer Inst 1993; 85(5):365-376.

24. Ware Junior JE, Sherbourne CD. The MOS 36-item short-form health survey (SF-36). I. Conceptual framework and item selection. Med Care 1992; 30(6):473-483.

25. The WHOQOL Group. The World Health Organization Quality of Life Assessment (WHOQOL): development and general psychometric properties. Soc Sci Med 1998; 46(12):1569-1585.

26. The WHOQOL Group. Development of the World Health Organization WHOQOL-B: quality of life assessment.1998; 28:55-58. 
27. Haes JC, Welvaart K. Quality of life after breas cancer surgery. J Surg Oncol 1985; 28(2):123-125.

28. Meyerowitz BE. Psychosocial correlates of breast cancer and its treatments. Psychol Bull 1980; 87(1):108-131.

29. Haes JC, Van Oostrom MA, Welwaart K. The effect of radical and conserving surgery on the quality of life of early breast cancer patients. Eur J Surg Oncol 1986; 12(4):337-342.

30. Haes JCJM, Pruyn JFA, Van Knippenberg FCE. Klachtenlijst voor kankerpatiënten, eerste ervaringen. Ned Tijdschr Psychol 1983; 38:402-408.

31. Trew M, Maguire P. Further comparison of two instruments for measuring quality of life in cancer patients. In: Beckman J, editor. Quality of life. Paris: Proc. Third EORTC Workshop on Quality of Life; 1982. p. 111.

32. Kemeny MM, Wellisch DK, Schain WS. Psychosocial outcome in a randomized surgical trial for treatment of primary breast cancer. Cancer 1988; 62(6):1231-1237.

33. Derogatis LR. Brief Symptom Inventory. Baltimore: Clinical Psychometric Research; 1975.

34. Skrzypulec V, Tobor E, Drosdzol A, Nowosielski K. Biopsychosocial functioning of women after mastectomy. J Clin Nurs 2009; 18(4):613-619.

35. Neugarten BL, Havighurst RJ, Tobin SS. The measurement of life satisfaction. J Gerontol 1961;16:134143.

36. Horowitz M, Wilner N, Alvarez W. Impact of event scale: a measure of subjective stress. Psychosom Med 1979; 41(3):209-218.

37. Zigmond AS, Snaith RP. The hospital anxiety and depression scale. Acta Psychiatr Scand 1983; 67(6):361370.

38. Snell WEJ, Papini D. The Sexuality Scale (SS): an instrument to measure sexual-esteem, sexual-depression, and sexual-preoccupation. J Sex Res 1989; 26(2):256-263.

39. Haes JC, Curran D, Aaronson NK, Fentiman IS. Quality of life in breast cancer patients aged over 70 years, participating in the EORTC 10850 randomised clinical trial. Eur J Cancer 2003; 39(7):945-951.

40. Chang JT, Chen CJ, Lin YC, Chen YC, Lin CY, Cheng AJ. Health-related quality of life and patient satisfaction after treatment for breast cancer in northern Taiwan. Int J Radiat Oncol Biol Phys 2007; 69(1):49-53

41. Ganz PA, Schag AC, Lee JJ, Polinsky ML, Tan SJ. Breast Conservation Versus Mastectomy: is there a difference in psychological adjustment or quality of life in the year after surgery? Cancer 1992; 69(7): 1729-1738.
42. Cella DF, Tulsky DS, Gray G, Sarafian B, Linn E, Bonomi A, Silberman M, Yellen SB, Winicour P, Brannon J. The functional assessment of cancer therapy scale: development and validation of the general measure. J Clin Oncol 1993; 11(3):570-579.

43. Schipper H, Clinch J, McMurray A, Levitt M. Measuring the quality of life of cancer patients: the functional living indexcancer: development and validation. J Clin Oncol 1984; 2(5):472-483.

44. Ganz PA, Schag CA, Cheng HL. Assessing the quality of life: a study in newly-diagnosed breast cancer patients. J Clin Epidemiol 1990; 43(1):75-86.

45. Schag CA, Heinrich RL, Aadland RL, Ganz PA. Assessing problems of cancer patients: psychometric properties of the cancer inventory of problem situations. Health Psychol 1990; 9(1):83-102.

46. Karnofsky DA, Burchenal JH. The clinical evaluation of chemotherapeutic agents in cancer. In: MacLeod CM, editors. Evaluation of chemotherapeutic agents. New York: Columbia University Press; 1949. p. 191-205.

47. McNair DM, Lorr M, Droppelman F. EITS manual for the profile of mood states. San Diego: Educational and Industrial Testing Service; 1971/1981.

48. Morrow GR, Feldstein M, Adler LM, Derogatis LR, Enelow AJ, Gates C, Holland J, Melisaratos N, Murawski BJ, Penman D, Schmale A, Schmitt M, Morse I. Development of brief measures of psychosocial adjustment to medical illness applied to cancer patients. Gen Hosp Psychiatry 1981; 3(2):79-88.

49. Thuler LCS, Mendonça GA. Estadiamento inicial dos casos de câncer de mama e colo do útero em mulheres brasileiras. Rev Bras Ginecol Obstet 2005; 27(11):656-660.

50. Parkes CM. Luto: estudos sobre a perda na vida adulta. São Paulo: Summus; 1998.

Apresentado em 22/09/2010

Aprovado em 05/01/2011

Versão final apresentada em 16/01/2011 